\title{
MEASUREMENT OF THE MAGNITUDE OF ForCE APPLIED BY STUDENTS WHEN LEARNING A MOBILISATION TECHNIQUE
}

\begin{abstract}
Passive accessory intervertebral movements (PAIVM's) are frequently used by physiotherapists in the assessment and management of patients. Studies investigating the reliability of passive mobilisation techniques have shown conflicting results. Therefore, standardisation of PAIVM's is essential for research and teaching purposes, which could result in better clinical management. In order to standardise graded passive mobilisation techniques, a reliable, easy-to-use, objective measurement tool must be used. The aim of this study was to determine whether it is necessary to quantify the magnitude of force applied when teaching a grade I central posteroanterior (PA) mobilisation technique (according to Mait-

\section{SMIT E, HonsBScPhysiotherapy ${ }^{1}$, W ESSELS J, B Ing, MSc Physiology ${ }^{3}$, W ITBO OI I, BScPhysiotherapy ${ }^{4}$, OTTO R, BSc Physiotherapy5.} CONRADIE M, HonsBScPhysiotherapy²,

1 Lecturer, Department of Physiotherapy, Faculty of Health Sciences, Stellenbosch University.

2 Lecturer, Department of Physiotherapy, Faculty of Health Sciences, Stellenbosch University.

3 Departement of Medical Physiology and Biochemistry, Faculty of Health Sciences, Stellenbosch University.

land) on the cervical spine. An objective measurement tool (FlexiForceTM) was used to determine the consistency of force applied by third and fourth year physiotherapy students while performing this technique. Twenty third-and 20 fourth year physiotherapy students $(n=40)$ were randomly selected. Each subject performed a grade I central PA on sensors placed on C6 for 25 seconds. The average maximum grade 1 force applied by the third year students was significantly higher than the force applied by the fourth year students $(p=0.034)$. There was a significantly larger variation in applied force among third years $(p=0.00043)$. The results indicate that the current teaching method is insufficient to ensure inter-therapist reliability amongst students, emphasising the need for an objective measurement tool to be used for teaching students. The measurement tool used in this study is economical, easily applied and is an efficient method of measuring the magnitude of force. Further research is needed to demonstrate the reliability and validity of the tool to assist teaching and research in a clinical setting.
\end{abstract}

KEY WORDS: MOBILISATION, POSTEROANTERIOR, FORCE-MEASUREMENT INSTRUMENT, INTER-THERAPIST RELIABILITY, STUDENT, LEARNING.

\section{INTRODUCTION}

Passive accessory intervertebral movements (PAIVM's) are frequently used by physiotherapists when assessing and managing patients with dysfunction of the spine. In orthopaedic manual therapy (OMT), a central posteroanterior (PA) mobilisation technique is valuable when assessing and treating cervical pain. The technique involves the application of an oscillatory force in a postero-anterior direction to the spinous process of the vertebra to be mobilised while the patient is positioned in prone (Maitland 1993). When testing movements by palpation techniques such as PAIVM's the passive movement of the one vertebra in relation to the adjacent vertebra must be appreciated to determine the presence of hypo- or hypermobility. These manual techniques form the basis for clinical reasoning regarding the diagnosis and the selection of techniques used to relieve pain, increase mobility and restore optimal function.

Maitland (1993) graded passive mobilisation techniques from grade I to IV, according to their relationship with the onset of perceived resistance (R1) and the size of the oscillations (amplitude). Grading provides a benchmark for the following: the selection of a specific treatment plan; the re-assessment of the effects of treatment techniques; teaching purposes; communication and encouragement of continual development of palpatory skills (Chester and Watson 2000). Maitland (1993) defined a grade I as a small-amplitude movement performed at the beginning of range without moving into resistance (R1). According to Maitland (1993, p.97) a fitting descrip- tion for a grade I PA pressure on the spinous process is to say that: "If a fly were between a therapist's thumbs and a spinous process it would not be squashed by the technique". He also stated that gentle techniques, such as a grade I, are particularly useful in the presence of severe pain, neurological changes or muscle spasm.

\section{CORRESPONDENCE TO:}

E Smit

Department of Physiotherapy,

Faculty of Health Sciences,

Stellenbosch University

PO Box 19063

Tygerberg 7505

Tel: (021) 9389300

Fax: (021) 9317810

E-mail: es6@sun.ac.za 
Based on the above it is clear that the magnitude of the applied force is determined by the therapist's perception of the onset of resistance (R1) to intervertebral movement. The question therefore arises whether physiotherapy students in their clinical years of training are able to demonstrate intra- and inter-therapist reliability when using graded passive mobilisation techniques.

PAIVM's are taught to physiotherapists during their education programmes and used in clinical practice and it is thus important to determine whether these techniques are reliable (Smedmark et al 2000). For a specific PAIVM to be reliable, safe and effective a number of variables relating to the manner of application of a manual force must fall within an accepted range of values (Task Force on Standards for Measurements in Physical Therapy 1991, Threlkeld 1992). The following variables were identified by Lee et al (1996): magnitude of applied force; direction of force; duration of loading; frequency of oscillation; specific vertebra and contact area to which the force is applied; and location of manual force in relation to the centre of the vertebra.

Studies investigating the reliability of passive mobilisation techniques have shown conflicting results (Smedmark et al 2000; Binkley et al 1995; Inscoe et al 1995; Simmonds et al 1995; Lindsay et al 1995). A study performed by experienced clinicians, investigating the mobility of three segments of the cervical spine and the first rib by passive physiological intervertebral movements (PPIVM's), demonstrated that interexaminer reliability was greater than expected by chance (Smedmark et al 2000). Intra-reliability (67 and 75\%) was better than inter-reliability (49\%) when assessing mobility by PPIVM's on 6 segments of the lumbar spine (Inscoe et al 1995). In the study by Binkley et al (1995), six experienced orthopeadic physical therapists performed PA accessory mobility testing on all levels of the lumbar spine, demonstrating poor inter-therapist reliability. Good interrater agreement $(88 \%)$ between two manual therapists were found regarding PA accessory glides on the sacro-illiac joint, opposed to poor reliability regard- ing central (14\%) and unilateral PA (46\%) on the lumbar spine. The lack of evidence on the specific magnitude of force required to perform the different 'grades of movements' makes generalisations about the reliability and validity of mobilisation techniques of limited value and prevents meaningful comparison between studies (Petty and Messenger 1996). If passive mobilisations are graded incorrectly and not standardised regarding the magnitude of force applied, it will have implications for both clinical practice and research studies. For objective investigation of the utilisation of PA mobilisation techniques, it is therefore necessary to accurately quantify the magnitude of force applied during the technique.

Several methods of measuring the magnitude of force applied during passive mobilisation on the spine have been described. Petty and Messenger (1996) developed the Kistler force platform that indirectly measured the vertical forces applied during a simulated PA mobilisation technique. The results were compared to the direct measurements of a Pinch Grip Analyser (PGA). However, in $90 \%$ of the trials, the force platform over-estimated and in $80 \%$ under-estimated the minimum and maximum peak forces applied to the PGA. Even though the force platform is considered a valid measurement tool, the accuracy is questionable. Whilst the force platform provides realistic estimates of the forces applied during the mobilisation technique, it must be recognised that this method does not allow the therapist to make contact with the treatment plinth during the execution of the technique. It therefore requires modifications of the mobilisation technique that therapists would use in normal clinical practise.

An instrument developed by Chester and Watson (2000), measured the magnitude of force by simulating natural spinal resistance using 4 sorbo-elastic balls (squash balls) on a construct model. This instrument has limitations in terms of validity and clinical application on a human body.

The equipment used in this study is based on an existing model (Jull and Bullock 1987), which is one of the earliest direct methods of determining the magnitude of force applied during a PA mobilisation using a thin capacitive pressure transducer. For the present study, the equipment has been modified using ultra-thin transducers.

It is important for any profession, including physiotherapy, to base their practice on the best available evidence. This implies that techniques chosen for patient management should have an acceptable level of validity and reliability. In order to make the use of PAIVM's more reliable, the magnitude of force applied to a real patient should be measured objectively, supplementing the qualitative descriptions of force, so that standardisation can be achieved. This study determines whether it is necessary to quantify the magnitude of force applied when teaching a grade I central PA mobilisation technique (according to Maitland) on C6.

\section{METHODOLOGY Equipment}

The force exerted by the left and right thumbs of the subjects was measured using two independent variable resistance force transducers (FlexiForce ${ }^{\mathrm{TM}}$, Tekscan, South Boston, MA). These ultra-thin $(0.13 \mathrm{~mm})$ transducers are sufficiently flexible and pliable to allow for minimally intrusive force measurement. The chosen sensor (Model A101) has a measuring range of $0-500$ gram. The sensor's active sensing area has a round footprint with a diameter of $10 \mathrm{~mm}$ which is large enough to measure the force exerted by the thumb and yet small enough to be placed side by side on the vertebra. Applying force on the sensing area results in a change in resistance of the sensor. An amplifier circuit was designed to convert the applied force to a voltage signal. The amplifier gain was adjusted so that a force between 0 and 500 gram was converted to voltage signals ranging from 0-10 Volt. The amplified voltage signals were digitized at a rate of $100 \mathrm{~Hz}$ using a multi-channel analogto-digital converter, which was lugged into a $486 \mathrm{DX} 2-66 \mathrm{MHz}$ personal computer (PC). A software package was written in Turbo Pascal to capture, process and display the force data instantly. The captured data was also stored to disk for post-processing. 
A special calibration jig was designed to calibrate the FlexiForce ${ }^{\mathrm{TM}}$ sensors. The calibration procedure consisted of progressively loading the sensor over the entire range of 0 to 500 gram using 50 gram increments. A calibration curve was obtained through fitting a linear regression line through the data using customized MathCad 8 (MathSoft Inc, Cambridge MA) routines. A two-point calibration check was performed before each session. The sensors remained stable over the two-week test period.

\section{Methods and procedure}

Twenty third year and 20 fourth year physiotherapy students at Stellenbosch University, South Africa, were randomly selected. All students were included for sampling except those repeating their third or fourth year of studies respectively. Measurements were taken after graded passive mobilisation techniques had been taught in the third year curriculum prior to clinical commencement. A pilot study was conducted on 5 qualified physiotherapists prior to testing and no changes were necessary. A 20-yearold asymptomatic female with no previous medical history of cervical symptoms gave consent to be the model for the duration of the study. The testing protocol was described to each subject and informed consent was obtained.

The following protocol was followed:

1. The model was positioned in prone on a cut-away plinth. The researchers ensured a neutral cervical spine and placed and secured the sensors over the model's C6 spinous process prior to each trial.

2. Third and fourth year students performed grade I central PA oscillations in a random order to eliminate any possible change in vertebral mobility.

3. The student's standing position and the positioning of each thumb on a sensor was standardized. A step-up bench was provided if requested by subjects.

4. Each student was allowed 30 seconds to feel or 'gauge' grade I PA and become comfortable with the sensors. Thereafter, the measurement tool captured the magnitude of the oscillatory force while the student per- formed grade I central PA oscillations for 25 seconds. The oscillatory force was instantly displayed on the PCscreen, which was positioned in such a way that the force data was only visible to the researcher. The students received no visible or audible feedback while performing the procedure. Immediately after the measurements, each student gave feedback regarding their opinion of the possible influence of the sensors on the technique.

The procedure was performed by 3 third and 3 fourth year students per day with five-minute intervals. The tests were completed within a two-week period.

\section{Data Analysis}

Experimental data was analysed in an Excel spreadsheet. The first 15 peaks of the data from the subjects' right thumb were isolated and the maximum values determined using the built in $\max$ function.

\section{Statistical Analysis}

The individual values of the average maximum peak force were calculated for the third and fourth year student groups respectively to determine the difference in the magnitude of force applied during a grade I central PA between third and fourth years by using a one-tailed unpaired t-test $(\alpha=0.05)$. Only data of the right thumb was used for this analysis. The extremely small magnitude of force measured by the left thumb was disregarded because of its insignificance. The coefficient of variation for each student was calculated and these values were compared by using a one-tailed unpaired t-test $(\alpha=0.05)$ to determine the variation in applied force between the third and fourth year student groups. The Bland-Altman statistic was calculated to determine the measure of agreement between the third and fourth year students showing the range of difference between the two groups.

\section{RESULTS}

The average maximum peak force applied by third year students during a grade I central PA mobilisation technique is significantly greater than fourth year students $(\mathrm{p}=0.034)$ (Figure 1). The third year students had a significantly larger variation in the magnitude of the average peak force applied in comparison to the fourth year students $(\mathrm{p}=0.00043)$ (Figure 2).

The Bland-Altman statistical test (Figure 3) showed that the majority of both the third and fourth year students agreed on an average maximum peak force for a grade I central PA between 100g-200g (x-axis). The mean difference

Figure 1: Comparison of Magnitude of Applied Force.

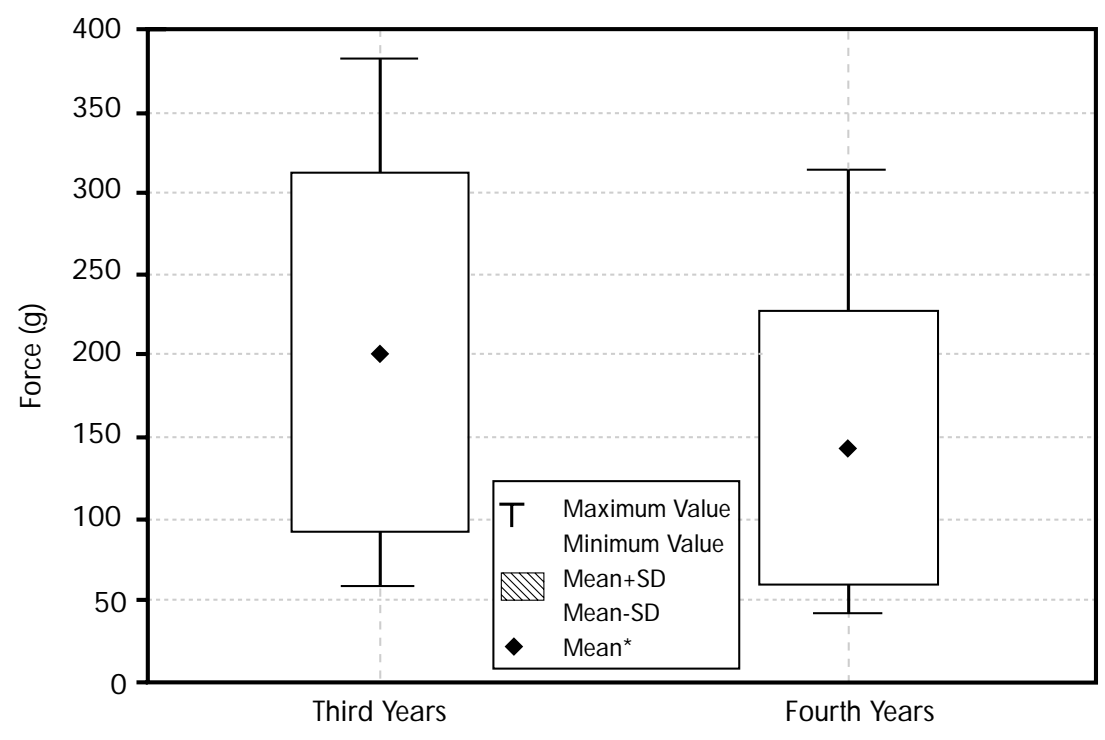


in maximum peak force between the third and fourth year students was $64 \mathrm{~g}$ (solid black line, $y$-axis). Most of the data points lie above the zero difference line indicating a positive difference between the two groups, showing that the maximum peak force applied by the third years students was between $100 \mathrm{~g}-300 \mathrm{~g}$ (area between dotted lines, y-axis) greater than the fourth year students.

The students' feedback about the influence of the sensors on the technique indicated that 11 subjects $(27.5 \%)$ reported a slight disturbance in tactile feedback, however, without a probable influence on the grading and the technique itself. Twenty-nine students (72.5\%) found that the sensors had no influence.

\section{DISCUSSION}

This study showed poor inter-therapist reliability regarding the magnitude of force applied by third and fourth year physiotherapy students during performance of a grade I central PA on C6. The third years applied significantly greater force and showed a greater variation

\section{Figure 2: Comparison of the Coefficient of Variation.}

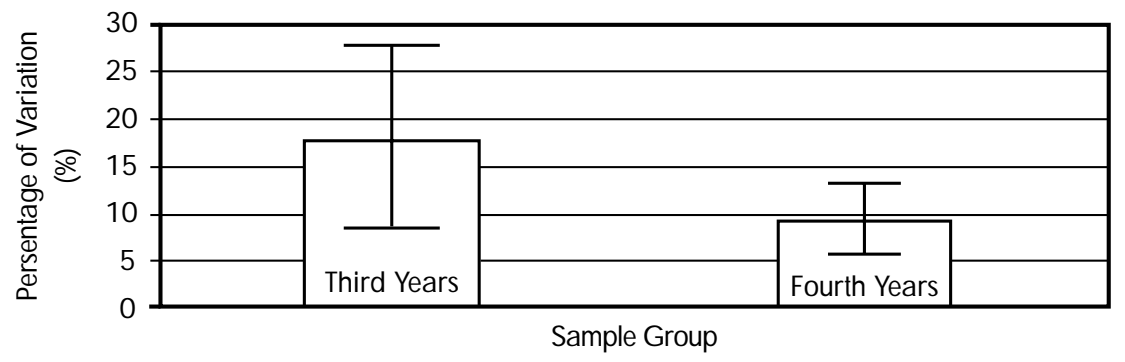

Figure 3: Measure of Agreement.

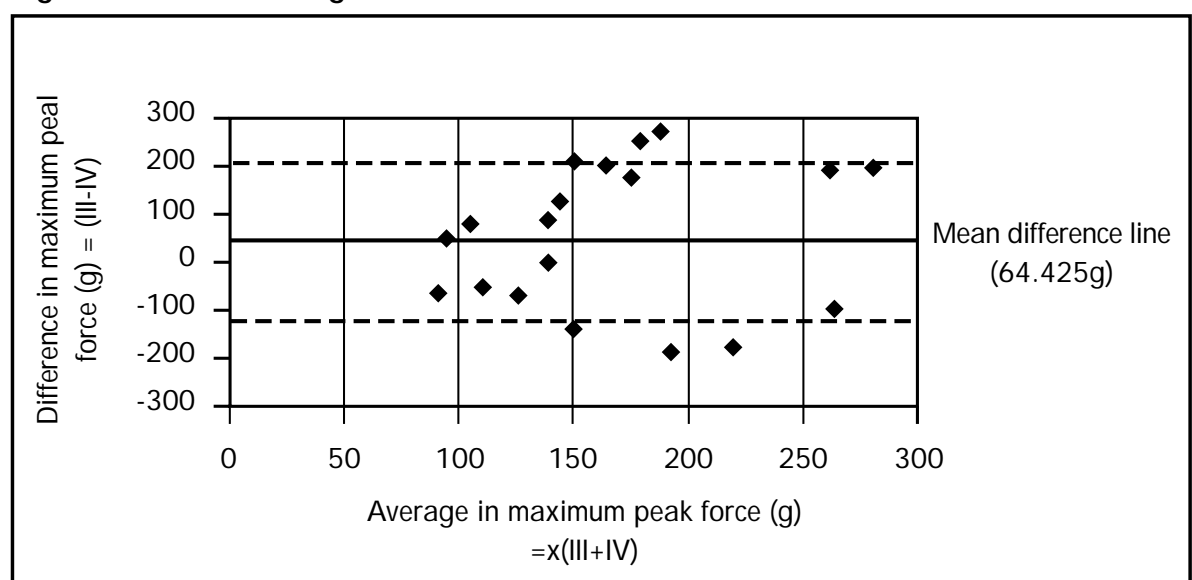

Maitland (1993) graded passive mobilisation techniques from grade I to IV, according to their relationship with the onset of perceived resistance. A possible explanation for the poor agreement amongst the third year students may relate to the inability to accurately detect the onset of resistance (R1) during the central PA mobilisation (Petty et al 2002). The results suggest that particularly third year students performed the 'grade I' technique into resistance. Thus, the technique was no longer a grade I, but a grade III or even a grade IV. The better agreement amongst fourth year students may be due to an extra year of clinical and practical experience, which could lead to the refinement of the palpatory skill of tactile feedback during passive intervertebral accessory movements (PAIVM's) and when determining R1. However, since PA mobilisation techniques are learnt in the third year, less variation amongst this group would be expected regarding the magnitude of applied force. Thus, it seems that a sound theoretical basis without practical application is not enough to ensure reliability.

Assessment of mobility between two vertebrae is difficult and the motor skill requires specific education and experience (Jull et al 1988). The learning of motor skills require several factors, including an explanation, repetition, as well as extrinsic verbal and visual feedback in order to make the necessary corrections (Shumway-Cook and Woollacott 1995). The following training method for the teaching of mobilisation techniques is used at Stellenbosch University: theoretical explanation based on Maitland's qualitative definition, accompanied by a practical demonstration, followed by supervised practice of that skill on fellow students. Ongoing verbal feedback from fellow students and lecturers is based on the perceived magnitude of force that should be applied and therefore to be subjective.

The results of this study indicate that the current teaching method of this technique did not ensure inter-therapist reliability amongst students. The qualitative definition of grades of movement that is taught to students is dependent on the palpation of R1. As the detection of 
R1 is crucial for grading, students need to develop fine palpatory skills to detect R1 and perform the different grades of movement accurately. According to Threlkeld (1992) it is important to standardise the magnitude of force applied during the different grades of movement to ensure that a PAIVM is reliable, safe and effective.

If the magnitude of force applied during a grade I central PA on a particular model, can be established by a qualified physiotherapist, with experience in OMT, the measured force can be used as a teaching aid for students to learn the motor skill. This can be expanded to the palpation of R1, other grades of movement, performed on different levels of the spine, and during different passive mobilisation techniques. A quantitative measurement tool with instant audiovisual feedback might enable students to perform the different grades of movement more accurately and master their techniques sooner. If inter-therapist reliability amongst students can be improved during the teaching of mobilisation techniques, this will have implications for more effective clinical practice.

\section{Measurement tool}

In this study the magnitude of force applied during a grade I central PA was quantified by a measurement tool. This tool can provide objective feedback to students for teaching purposes. Even though other tools are available, this tool was tested on a human model which is in contrast to the studies on the Force platform (Petty and Messenger 1996) and Spinal Mobilization Model (Simmonds et al 1995). The tool used in this study has the additional facility to give instant visual feedback on magnitude of force, amplitude and frequency of oscillation while the technique is being performed. According to Lee et al (1990) simultaneous visual feedback will result in immediate improvement in the accuracy and consistency whereby passive mobilisation techniques are being performed. Using this objective measurement tool, with the facility of simultaneous visual feedback, student learning can be assisted because immediate adaptations to techniques can be made. This can only improve inter-therapist reliability on the magnitude of force used during different grades of movement.

Additional attributes of the tool used in this study are that it is an inexpensive, compact unit, which is easily set up, transported and stored. The computer programme is uncomplicated and data is available instantly. The sensors proved to be highly durable since the same sensors could be used throughout this study. The small size and the versatility of the sensors make placement thereof easy. The sensors are very sensitive and accurately measure very low forces in grams whereas the other studies done by Chester and Watson (2000) as well as Petty and Messenger (1996) measured forces ranging in kilograms. Therefore, this tool is most suitable for measuring the low forces applied during grade I mobilisation techniques.

In this study, the students' feedback regarding the influence of the sensors on their performance of a grade I central PA technique, indicated that $72.5 \%$ of the students reported no interference, while $27.5 \%$ of the students reported a slight disturbance of tactile feedback without probable influence on the grading and the technique itself. Chester and Watson (2000) and Petty and Messenger (1996) stated that a tool that uses pressure sensors will interfere with the palpation of soft tissues, especially at the beginning of range. The authors of this study suggest that the problem of contacting the spinous process through sensors overlying it, may be resolved to some degree by customizing the sensor material to restore some proprioception which is lost while performing the technique.

The sensors chosen for this study saturate at 500 grams and any force value above that cannot be recorded. Five third year students and one fourth year student performed the technique with forces exceeding $500 \mathrm{~g}$, resulting in loss of data. The range of forces that can be measured by the tool can however be adjusted by changing the sensors. It is therefore recommended that the sensors could be changed to ensure that larger forces could also be measured in future studies. This objective tool can therefore be made suitable for research on grades II, III and IV where greater forces are required.
If graded passive mobilisation techniques like PAIVM's are performed with more reliability during teaching, there could be various clinical, financial and research implications. Clinically, physiotherapists would be able to assess patients more precisely, treat patients more effectively and safely, utilizing more reliable outcomes to ensure outcomebased practice. This will also have financial implications, benefiting the patient, medical aids and state funding. Research will be aided due to objective measurements of applied forces during PAIVM's and better standardisation of mobilisation techniques so that results of different studies can be compared.

\section{CONCLUSION}

A poor inter-therapist reliability in the magnitude of force applied during a PAIVM was found in third and fourth year physiotherapy students. Third years applied significant higher forces during the performance of a grade I central PA mobilisation technique, and fourth years were in better agreement with each other in terms of magnitude of force applied. This emphasizes that current teaching methods are inadequate, especially in the third year of training, when student clinical practice commences. Training in PAIVM's with audio-visual feedback, either instantly or after performing the technique, could improve the consistency and accuracy of applied forces, consequently improving the standard of reliability of these techniques. If standardisation and reliability of techniques can be improved sooner during teaching, the quality of research and ultimately outcome-based client management will also improve.

Further research is needed to demonstrate the reliability and validity of this tool to assist teaching and research in a clinical setting. With minor modifications, the tool used in this study to quantify the magnitude of force applied during a grade I central PA mobilisation technique, (according to Maitland 1993) could also be utilized for the detection of $\mathrm{R} 1$, as well as magnitude of forces applied during grade II, III and IV. 


\section{REFERENCES}

Binkley J, Stratford PW, Gill C 1995 Interrater reliability of lumbar accessory motion mobility testing. Physical Therapy 75(9): 786-792

Chester R, Watson MJ 2000 A newly developed spinal simulator. Manual Therapy 5(4): 234-242

Inscoe EL, Witt PL, Gross MT, Mithell RU 1995 Reliability in evaluating passive intervertebral motion of the lumbar spine. The Journal of Manual and Manipulative Therapy 3(4): 135-143

Jull G, Bullock M 1987 A motion profile of the lumbar spine in an ageing population assessed by manual examination. Physiotherapy Practice 3: 70-81

Jull G, Bogduk N, Marsland 1988 The accuracy of manual diagnosis for cervical zygaphophysial joint pain syndromes. The Medical Journal of Australia 148: 233-236
Lee M, Moseley A, Refshauge 1990 Effect of feedback on learning a vertebral joint mobilization skill. Physical Therapy 70(2): 97-104

Lee M, Steven GP, Crosbie J, Higgs RJED 1996 Toward a theory of lumbar mobilisation - the relationship between applied manual force and movements of the spine. Manual Therapy 2: 67-75

Lindsay DM, Meeuwisse WH, Mooney ME, Summersides J 1995 Interrater reliability of manual therapy assessment techniques. Physiotherapy Canada 47(3): 173-179

Maitland GD 1993 Vertebral Manipulation 5th edn. Butterworth-Hienemann, Boston

Petty NJ, Maher C, Latimer J, Lee M 2002 Manual examination of accessory movements - seeking R1. Manual Therapy 7(1): 39-43

Petty NJ, Messenger N 1996 Can the force platform be used to measure the forces applied during a PA mobilisation of the lumbar spine?
The Journal of Manual and Manipulative Therapy 4(2): 70-76

Shumway-Cook A, Woollacott M 1995 Motor control, theory and practical applications, pp 33-37. Williams and Wilkins, Baltimore.

Simmonds MJ, Kumar S, Lechelt E 1995 Use of a spinal model to quantify the forces and motion that occur during therapists' tests of spinal motion. Physical Therapy 75(3): 212-222

Smedmark V, Wallin M, Arvidsson I 2000 Inter-examiner reliability in assessing passive intervertebral motion of the cervical spine. Manual Therapy 5(2): 97-101

Task Force on Standards for Measurement in Physical Therapy 1991 Standards for tests and measurements in physical therapy practice. Physical Therapy 71(8): 589-597

Threlkeld AJ 1992 The effects of manual therapy on connective tissue. Physical Therapy 172(12): 893-901

\begin{tabular}{|c|c|c|}
\hline \multicolumn{3}{|c|}{$\begin{array}{c}\text { Platinum Health } \\
\text { Rustenburg } \\
\text { Vacancy - Physiotherapist } \\
\text { A vacancy currently exists at Platinum Health } \\
\text { in Rustenburg for a Physiotherapist. }\end{array}$} \\
\hline \multicolumn{3}{|c|}{$\begin{array}{l}\text { Selection Criteria. } \\
\text { - Relevant degree/diploma in Physiotherapy. } \\
\text { - Must have } 2 \text { years relevant experience. } \\
\text { - Must be computer literate } \\
\text { - Registered with Health Professional Council of SA. }\end{array}$} \\
\hline \multicolumn{3}{|c|}{$\begin{array}{l}\text { The position offers the following: } \\
\text { - Market related salary } \\
\text { - Provident Fund } \\
\text { - Medical Aid }\end{array}$} \\
\hline \multicolumn{3}{|c|}{$\begin{array}{l}\text { Interested candidates are invited to forward a detailed Curriculum } \\
\text { Vitae to: Attention: HR M anager }\end{array}$} \\
\hline & $\begin{array}{l}\text { Fax: } 014-5942820 \\
\text { Closing date for app }\end{array}$ & $\begin{array}{l}\text { e-mail: pauldp@angloplats.com } \\
\text { ons is } 21 \text { N ovember } 2003\end{array}$ \\
\hline \multicolumn{3}{|c|}{$\begin{array}{l}\text { Platinum Health shall apply affirmative action principles as set out } \\
\text { in the company's employment equity act. }\end{array}$} \\
\hline \multicolumn{3}{|c|}{$\begin{array}{l}\text { In the event you do not hear from the Company within a period } \\
\text { of } 21 \text { days after the closing date of applications, your application } \\
\text { shall be deemed to be unsuccessful. The CV's of unsuccessful } \\
\text { applications will not be returned. }\end{array}$} \\
\hline
\end{tabular}

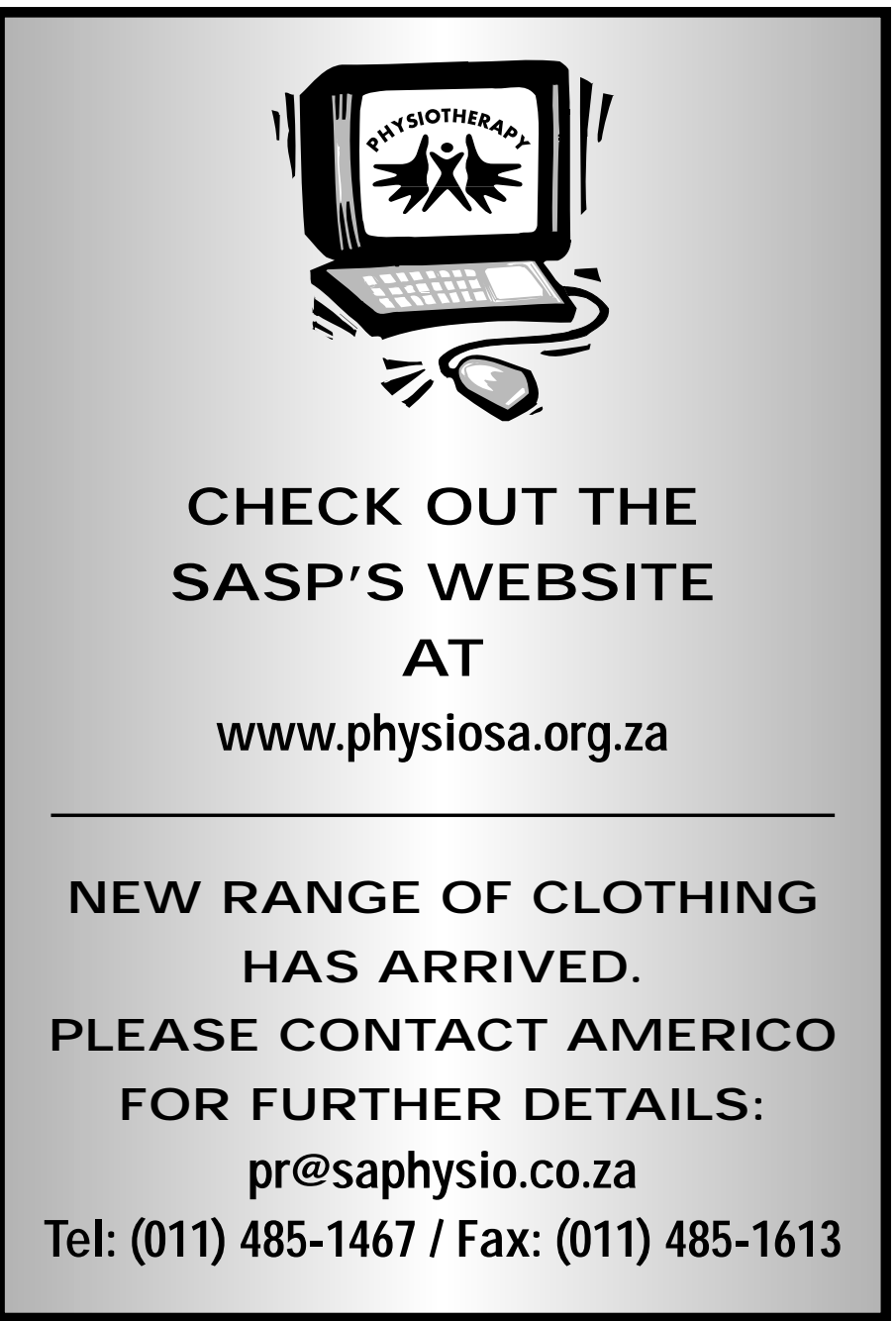

